\title{
COMMENTARY
}

\section{Invasive Ductular Reaction}

\section{Form and Function}

David B. Wilson ${ }^{* \dagger}$ and David A. Rudnick ${ }^{* \dagger}$

From the Department of Pediatrics, * Washington University School of Medicine, St. Louis Children's Hospital, St. Louis; and the Department of Developmental Biology, ${ }^{\dagger}$ Washington University School of Medicine, St. Louis, Missouri

The term ductular reaction (DR) was coined by Hans Popper in 1957 and refers to the histopathological expansion of epithelial cells, and associated inflammatory cells, stroma and other components, arising at the hepatocellular-biliary boundary in the setting of acute severe hepatocellular injury or chronic hepatocellular or biliary obstructive liver diseases. ${ }^{1-3}$ Even before Popper's report, descriptions of this phenomenon are purported to have appeared in the medical literature dating as far back as the mid-19th century. ${ }^{2}$ Recent renewed interest in DR has been prompted by investigations implicating this process as a mediator of progenitorcell-based regenerative recovery of the liver from severe injury. ${ }^{4}$ However, the functional and translational significance of this phenomenon remains uncertain. ${ }^{5,6}$ The research article by Clerbaux et $\mathrm{al}^{7}$ in this issue of The American Journal of Pathology (AJP) provides novel insights into this long-recognized, but still relatively poorly understood, response to severe and chronic liver injury.

DR occurs in association with many forms of human liver disease and in numerous experimental animal models of liver injury. ${ }^{2-5}$ Distinct histopathological DR subtypes have been recognized to occur in different settings and described using various nomenclatures (Table 1). For example, the terms typical and atypical ductular proliferation have been used to distinguish the human DR histology associated with extrahepatic versus intrahepatic cholestasis, respectively. ${ }^{8}$ In this older classification scheme, typical ductular proliferation, which occurs in the setting of total extrahepatic biliary obstruction, arises from the proliferation of pre-existing ductules, and the associated DR is confined within the portal mesenchyme. In contrast, atypical ductular proliferation, as occurs as part of the regenerative response to hepatic necrosis, is characterized by distinct structures that extend beyond the portal mesenchyme into the hepatic lobule, and this response is closely associated with progenitor cell activation. Desmet's recognition of biliary metaplasia of hepatocytes in some cases of DR led to further distinction of DR subtypes into three categories, including: type 1, ie, typical DR; type 2, ie, atypical DR with ductular metaplasia of hepatocytes; and type 3, ie, atypical DR associated with progenitor cell proliferation (Table 1). ${ }^{2,4}$ This more comprehensive stratification has subsequently been adopted by an international group of liver pathologists and hepatologists as preferred over the older nomenclature. ${ }^{8}$ As already mentioned, the ensuing recognition that the atypical type 3 DR might contribute to hepatocellular replenishment in severe hepatocellular diseases led to initial enthusiasm for and further investigations of this possibility in various experimental models. However, the results of those studies have been conflicting, with some showing DRbased reconstitution of liver when hepatocellular regenerative capacity is significantly compromised or hepatic parenchymal injury is massive, ${ }^{9,10}$ and others revealing that when hepatocytes retain their regenerative potential the DR contribution to recovery of liver parenchyma is minimal. ${ }^{11,12}$ Thus, the functional relevance of DR in experimental and human liver disease remains ambiguous. ${ }^{5,6}$

In their report in this issue of $A J P{ }^{7}$ Clerbaux et al use lineage tracing and/or immunostaining to distinguish between noninvasive, minimally invasive, and invasive DR patterns (Table 1) in various mouse models and human liver biopsies of either primary hepatocellular or primary biliary

Accepted for publication June 4, 2019.

Disclosures: None declared.

Address correspondence to David A. Rudnick, M.D., Ph.D., Washington University School of Medicine, Box 8208, 660 S. Euclid Ave., St. Louis, Missouri 63110. E-mail: rudnick_d@wustl.edu 
Table 1 DR Categorization Scheme

\begin{tabular}{|c|c|c|}
\hline Older nomenclature $^{8}$ & Desmet $^{2,4}$ & Clerbaux $^{7}$ \\
\hline $\begin{array}{l}\text { Typical - arising from pre-existing ductules and } \\
\text { confined to the portal mesenchyme }\end{array}$ & Type 1 - typical DR & Non-invasive - typical DR \\
\hline $\begin{array}{l}\text { Atypical - characterized by structures extending } \\
\text { beyond the portal mesenchyme and associated } \\
\text { with progenitor cell activation }\end{array}$ & $\begin{array}{l}\text { Type } 3 \text { - atypical DR with progenitor cell } \\
\text { proliferation }\end{array}$ & $\begin{array}{l}\text { Invasive }- \text { DR that expands }>80 \\
\mu \mathrm{m} \text { beyond portal mesenchyme }\end{array}$ \\
\hline
\end{tabular}

obstructive liver injury and disease. As in the case of both typical DR and Desmet's type 1 DR, Clerbaux's noninvasive DR is defined by its confinement within the portal mesenchyme. Similarly, like atypical DR and Desmet's type 2- and type 3-DR's, minimally invasive and invasive DR expand beyond the portal mesenchyme border into the hepatic lobule. However, in contrast to Desmet's classification scheme, which incorporates histopathological features of hepatocellular metaplasia or progenitor cell expansion to define DR types 2 and 3, respectively, Clerbaux's categorization of minimally invasive versus invasive DR is delineated as such based on careful quantification of DR expansion beyond the portal mesenchyme border into the hepatic parenchyma (Table 1). Through painstaking morphometric analysis, using their previously reported OPN-iCre-ER ${ }^{T 2}$; Rosa26-YFP model in which cholangiocyte-derived cell populations are lineage-traced using a tamoxifen-inducible YFP reporter, ${ }^{11}$ the authors garner evidence to support the conclusions that invasive DR occurs in mice subjected to the choline-deficient, ethionine-
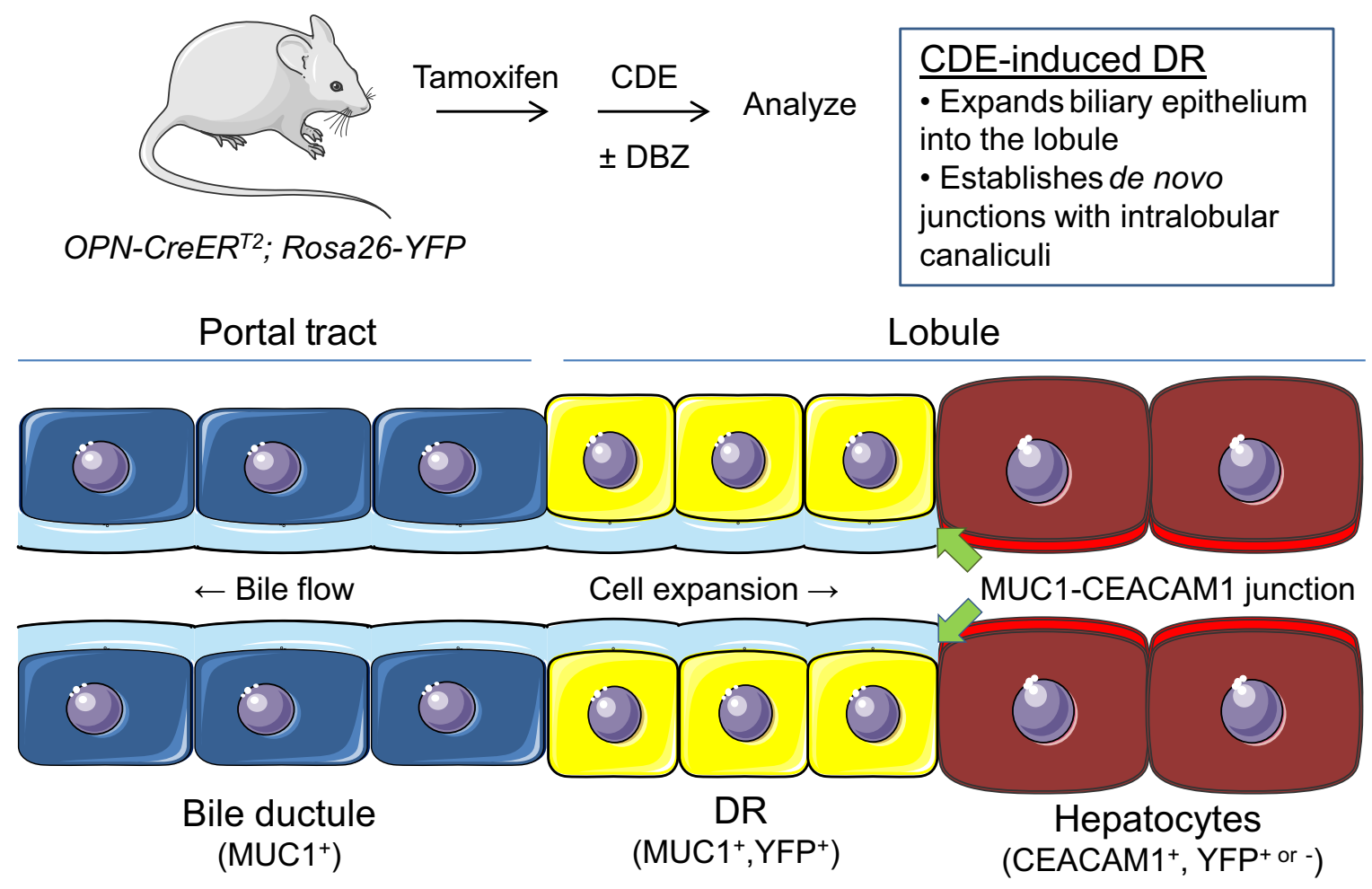

Figure 1 Induction of invasive DR promotes generation of de novo hepatobiliary junctions following severe hepatocellular injury in mice. In this edition of the American Journal of Pathology, Clerbaux et $\mathrm{al}^{7}$ use a tamoxifen-inducible Cre (OPN-CreER $\left.{ }^{T 2}\right)$ and Rosa26-YFP reporter to indelibly label DR cells and their descendants. Immunostaining of apical surface markers [MUC1 (pale blue), CEACAM1 (red)] allows bile ductules and DR cells to be distinguished from hepatocytes. The authors show that invasive DR, induced by exposure to a choline-deficient ethionine-supplemented (CDE) diet, establishes new junctions with intralobular canaliculi (green arrows) thereby maintaining structural continuity between the principal site of bile secretion, canaliculi, and the biliary tree. They also show that this process is attenuated by dibenzazepine (DBZ), an inhibitor of NOTCH signaling. Prepared using adaptations of image vectors from Servier Medical Art (www.servier.com, last accessed May 13, 2019), licensed under the Creative Commons Attribution 3.0 Unported License (https:// creativecommons.org/licenses/by/3.0). CDE, choline deficient ethionine-supplemented; CEACAM1, carcinoembryonic antigen related cell adhesion molecule1; DBZ, dibenzazepine; DR, ductular reaction; MUC1, mucin-1; OPN, Osteopontin; YFP, yellow fluorescent protein. 
supplemented diet (CDE) model of hepatocellular injury, and, moreover, that this CDE-induced invasive DR is associated with the establishment of new canalicular junctions between biliary/DR-derived cells and lobular hepatocytes (Figure 1). Although other strategies to lineage-trace biliary epithelium have been described, including reports based on Sox9- and cytokeratin-19-promoter driven CRE recombinase expression, ${ }^{13}$ the results of the approach used by Clerbaux et al are particularly compelling because of this group's previous demonstration of the specificity of osteopontin $(O P N)$-promoter-regulated reporter expression in cholangiocytes in the canals of Hering, intralobular ductules, and interlobular ducts, but no other liver cells in adult mice. ${ }^{11}$ These investigators also show that inhibition of NOTCH signaling impairs CDE-induced invasive DR and the establishment of de novo lobular duct-canalicular junctions, then go on to demonstrate that de novo lobular ductcanalicular junctions, which are detectable in the setting of CDE-induced invasive DR, are not detectable in mouse models of biliary obstruction, including bile-duct-ligation (BDL) and exposure to a 3,5-diethoxycarbonyl-1,4dihydrocollidine-(DDC)-containing diet. Finally, they provide additional data indicating that features of invasive DR associated with lobular hepatobiliary junctions are much more commonly detected in human liver biopsies from subjects with primary hepatocellular diseases compared to those with primary biliary liver disease. The investigators conclude that invasive DR plays a role in re-establishing structural and functional continuity between lobular hepatocytes and bile ducts after severe hepatocellular injury in mice and humans. Together, these findings provide a persuasive alternative to hepatocellular replenishment as the functional role of invasive DR in severe hepatocellular injury, ie, restoration of the injury-induced compromise in canalicular-ductal communication.

As novel and provocative as these findings and their corresponding implications are, the data also raise important new questions that can, and should now be addressed. For example, does invasive DR occur in other models of chronic hepatocellular injury and does it eventually even occur in the setting of biliary obstruction? If either is the case, are those responses similarly dependent upon NOTCH signaling? In the studies reported here, disruption of NOTCH signaling suppressed CDE-induced invasive DR. Nevertheless, HIPPO/YAP, WNT//-CATENIN, HGF/cMET, and other signaling pathways have also been identified as possible mediators of DR-associated cholangiocyte proliferation, cellular transdifferentiation, and/or progenitor cell expansion. ${ }^{14}$ Additionally, the zinc-finger transcription factor GATA6 was recently implicated as both a novel marker and putative inducer of DR in extrahepatic biliary atresia. ${ }^{15}$ Together, these considerations raise the intriguing question as to whether liver injury- and/or DR pattern-specific molecular mechanisms mediate invasive versus noninvasive DR, a question that can now be answered using experimental approaches analogous to those used here for NOTCH signaling. Gain-of-signaling-function effects could similarly be explored with such approaches to further define the molecular mechanisms that mediate DR. Finally, the histomorphometric and temporal relationships between Clerbaux's canalicular junction-establishing invasive DR and Desmet's progenitor cell proliferation-associated type 3 DR may also be examined in these paradigms. Longer term studies to address this consideration would reveal whether de novo lobular junctions remain lobular or, alternatively, if progenitor cell proliferation-dependent or some other sort of remodeling occurs over time. Though perhaps more challenging to address, such studies should also assess whether the presence or absence of invasive and/or type 3 DR predicts the well-known longer term risks of chronic liver diseases including cirrhosis and cancer in these animal models. Those results could have important clinical-translational implications for human liver diseases. Such queries are now both feasible and worthy of consideration based on the novel findings and provocative implications of the data reported in this manuscript.

\section{Acknowledgments}

We thank Marjut Pihlajoki and Markku Heikinheimo for their assistance with preparation of the figure and critical review of this commentary.

\section{References}

1. Popper H, Kent G, Stein R: Ductular cell reaction in the liver in hepatic injury. J Mt Sinai Hosp N Y 1957, 24:551-556

2. Desmet V, Roskams T, Van Eyken P: Ductular reaction in the liver. Pathol Res Pract 1995, 191:513-524

3. Williams MJ, Clouston AD, Forbes SJ: Links between hepatic fibrosis, ductular reaction, and progenitor cell expansion. Gastroenterology 2014, 146:349-356

4. Desmet VJ: Ductal plates in hepatic ductular reactions. Hypothesis and implications. I. Types of ductular reaction reconsidered. Virchows Arch 2011, 458:251-259

5. Roskams T, Desmet V: Ductular reaction and its diagnostic significance. Semin Diagn Pathol 1998, 15:259-269

6. Roskams T: Progenitor cell involvement in cirrhotic human liver diseases: from controversy to consensus. J Hepatol 2003, 39:431-434

7. Clerbaux LA, Manco R, Van Hul N, Bouzin C, Sciarra A, Sempoux C, Theise ND, Leclercq IA: Invasive ductular reaction operates hepatobiliary junctions upon hepatocellular injury in rodents and humans. Am J Pathol 2019, 189:1569-1581

8. Roskams TA, Theise ND, Balabaud C, Bhagat G, Bhathal PS, BioulacSage P, Brunt EM, Crawford JM, Crosby HA, Desmet V, Finegold MJ, Geller SA, Gouw AS, Hytiroglou P, Knisely AS, Kojiro M, Lefkowitch JH, Nakanuma Y, Olynyk JK, Park YN, Portmann B, Saxena R, Scheuer PJ, Strain AJ, Thung SN, Wanless IR, West AB: Nomenclature of the finer branches of the biliary tree: canals, ductules, 
and ductular reactions in human livers. Hepatology 2004, 39: $1739-1745$

9. Raven A, Lu WY, Man TY, Ferreira-Gonzalez S, O’Duibhir E, Dwyer BJ, Thomson JP, Meehan RR, Bogorad R, Koteliansky V, Kotelevtsev Y, Ffrench-Constant C, Boulter L, Forbes SJ: Cholangiocytes act as facultative liver stem cells during impaired hepatocyte regeneration. Nature 2017, 547:350-354

10. He J, Lu H, Zou Q, Luo L: Regeneration of liver after extreme hepatocyte loss occurs mainly via biliary transdifferentiation in zebrafish. Gastroenterology 2014, 146:789-800.e8

11. Espanol-Suner R, Carpentier R, Van Hul N, Legry V, Achouri Y, Cordi S, Jacquemin P, Lemaigre F, Leclercq IA: Liver progenitor cells yield functional hepatocytes in response to chronic liver injury in mice. Gastroenterology 2012, 143:1564-15675.e7
12. Schaub JR, Malato Y, Gormond C, Willenbring H: Evidence against a stem cell origin of new hepatocytes in a common mouse model of chronic liver injury. Cell Rep 2014, 8:933-939

13. Rubio-Tomas T, Aguilar-Bravo B, Sancho-Bru P: Genetic Lineage Tracing of Biliary Epithelial Cells. Methods Mol Biol 2019, 1905 : $45-57$

14. Sato K, Marzioni M, Meng F, Francis H, Glaser S, Alpini G: Ductular Reaction in Liver Diseases: pathological Mechanisms and Translational Significances. Hepatology 2019, 69:420-430

15. Soini T, Pihlajoki M, Andersson N, Lohi J, Huppert KA, Rudnick DA, Huppert SS, Wilson DB, Pakarinen MP, Heikinheimo M: Transcription factor GATA6: a novel marker and putative inducer of ductal metaplasia in biliary atresia. Am J Physiol Gastrointest Liver Physiol 2018, 314:G547-G558 